\title{
Shape Stability of Particleboards Covered with Decorative Veneers
}

\section{Stabilnost oblika ploča iverica površine obrađene dekorativnim furnirima}

\author{
Original scientific paper - Izvorni znanstveni rad \\ Received-prispjelo: 4. 7. 2012. \\ Accepted-prihvaćeno: 21. 6. 2013. \\ $U D K: 630 * 852.32 ; 630 * 832.281 ; 630 * 863.2$ \\ doi:10.5552/drind.2013.1234
}

\begin{abstract}
This paper summarizes the results of research aimed at determining the shape stability, modulus of rupture (MOR) and modulus of elasticity (MOE) of components made of particle boards, covered on their front side with decorative veneer of American walnut (Juglans nigra), $0.6 \mathrm{~mm}$ thick, and on the underside with decorative veneers of other species (Picea abies and Fagus sylvatica), 0.6-1.5 mm thick, or by a countermove foil of $85-90 \mathrm{~g} / \mathrm{m}^{2}$ surface weight. Subsequently, measurements were carried out of the shape stability (warping) of test specimens cut from various combinations of surface-finished boards. These test specimens were air conditioned in three different environments. At the same time, values were determined of bending strength and modulus of rupture with respect to the direction of fibres of sheathing materials. It has been found that the lowest warping values were achieved with combinations consisting of American walnut of a thickness of $0.6 \mathrm{~mm}$ on the tight side and alder (Alnus glutinosa), $0.6 \mathrm{~mm}$ thick, on the underside.
\end{abstract}

Keywords: veneer, veneering, panels, warping, shape stability

SAŽETAK • U radu se prikazuju rezultati istraživanja s ciljem utvrđivanja stabilnosti oblika, modula loma (MOR) i modula elastičnosti (MOE) komponenti proizvedenih od iverice, koje su na vanjskoj strani furnirane dekorativnim furnirom američkog oraha (Juglans nigra) debljine 0,6 mm, a na unutarnjoj strani dekorativnim furnirom drugih vrsta (Picea abies i Fagus sylvatica) debljine 0,6 - 1,5 mm ili folijom površinske mase $85-90 \mathrm{~g} / \mathrm{m}^{2}$. Nakon toga provedena su mjerenja stabilnosti oblika (izvitoperenosti) uzoraka pripremljenih od ploča različitih kombinacija površinske obrade. Ispitni uzorci klimatizirani su u tri različita okruženja. U isto vrijeme utvrđene su $i$ vrijednosti čvrstoće na savijanje te modula loma s obzirom na smjer vlakanaca materijala kojim su iverice obložene. Utvrđeno je da su najniže vrijednosti izvitoperenosti izmjerene u ploča obrađenih furnirom američkog oraha debljine 0,6 mm na vanjskoj strani i furnirom johe (Alnus glutinosa) debljine 0,6 mm na unutarnjoj strani.

Ključne riječi: furnir, furniranje, ploče, savijanje, stabilnost oblika

\section{INTRODUCTION}

\section{UVOD}

Demand for aesthetically pleasing wood is high abroad. However, considerable costs for logging operations, certification, transport and limited supplies of ex- otic wood are the cause of increasing prices of input raw materials for the manufacture of decorative veneers. For these reasons, the production and application of modified veneers develops, which makes possible to use less attractive veneers particularly on hidden surfaces. At present time, manufacturers make great efforts to re-

\footnotetext{
${ }^{1}$ Authors are associate professor, associate professor, assistant and assistant at Mendel University, Faculty of Forestry and Wood Technology, Brno, Czech Republic.

${ }^{1}$ Autori su izvaredni profesor, izvanredni profesor, asistentica i asistent Mendelova Sveučilišta, Fakultet šumarstva i drvne tehnologije, Brno, Republika Češka.
} 
place expensive veneers on hidden surfaces by countermove materials. The function of these materials is to provide shape stability of furniture elements.

A basic question is which countermove material can be chosen among specific kinds of wood and what relationships exist between them.

Determination of these relationships will contribute to create suitable combinations of veneers to provide shape stability, which will enable the production of products of competitive advantages by means of decreasing production costs (Král and Hrázský, 2005)

Generally, warping is the undesirable shape deformation of elements. All manufacturers try to produce and supply shape-stable elements to customers. These elements keep their shape and dimensions at moisture-heat stress, which can occur both in manufacture and during storage, transport and use. An area element was examined, and it consisted of a particleboard as the bearing (supporting) part and two covering veneer layers or a countermove foil as one layer. From the aspect of shape stability, the main problem of veneered elements consists in different dimensional changes of materials occurring in particular layers. Particleboards swell by $0.2-0.3 \%$ of board dimensions at moisture changes of $1 \%$ in both main directions (Požgaj et al., 1997), while wood swelling ranges at higher values. Changes in dimensions of longitudinal, radial and tangential swelling are in a ratio of 1:10:20. According to Ugolev (1975), coefficients of swelling and shrinking of selected kinds of wood are shown in Table 1.

If the layers were not stuck (connected) together, moisture changes would become evident in the change of their dimensions. However, dimensional changes of decorative surface layers would be even larger. After connecting the layers by means of bonding in the pro-
Table 1 Coefficients of shrinking and swelling Tablica 1. Koeficijenti utezanja i bubrenja

Coefficients of shrinking $\boldsymbol{\beta}$ and swelling $\boldsymbol{\alpha}$, $\% / \mathbf{1 \%}$ moisture / Koeficijenti utezanja $\beta$ i bubrenja $\alpha$, $\% / 1 \%$ sadržaja vode

\begin{tabular}{|c|c|c|c|c|}
\hline & \multicolumn{2}{|c|}{$\begin{array}{c}\text { Radial } \\
\text { Radijalno }\end{array}$} & \multicolumn{2}{c|}{$\begin{array}{c}\text { Tangential } \\
\text { Tangencijalno }\end{array}$} \\
\hline kind of wood / Vrsta drva & $K_{\beta}$ & $K_{\alpha}$ & $K_{\beta}$ & $K_{\alpha}$ \\
\hline Spruce / smreka & 0.16 & 0.17 & 0.28 & 0.31 \\
\hline Beech / bukva & 0.17 & 0.18 & 0.32 & 0.35 \\
\hline
\end{tabular}

cess of hot pressing into one compact unit, the shift of layers of the composite material is prevented. At the same time, however, it is necessary to take into account that due to this firm connection, a certain stress occurs in the layers at moisture changes, which affects this sheet composite material. If the sum of planar static moments is equal to zero, then the element maintains its planeness. This condition is valid provided that the surface composite material is compiled (put together) symmetrically, i.e. using identical surface layers. In case the sum of planar static moments is not equal to zero, the element is deformed. Therefore, efforts are made to create a composite material, which is most consistent with the theory of veneering, i.e. to achieve a symmetrical lay-out of materials (Avramidis et al., 2011). At the application of countermove materials, it is necessary to replace a veneer by another material or another veneer of the same properties as the veneer used on the front part of the board. In this way, it is possible to find suitable countermove materials for specific kinds of decorative veneers (Šrajer, 2013). In order to provide the shape stability of elements, it is most important to maintain stable relative moisture and temperature of the environment. In case of using countermove foils, unbleached sulphate pulp impregnated

Table 2 Combination of veneers and foil

Tablica 2. Kombinacija furnira i folije

\begin{tabular}{|c|c|c|}
\hline \multicolumn{2}{|c|}{$\begin{array}{c}\text { Veneer and foil combination } \\
\text { Kombinacija furnira i folije }\end{array}$} \\
\hline $\begin{array}{c}\text { Tight side } \\
\text { Vanjska strana }\end{array}$ & $\begin{array}{c}\text { Underside (countermove side) } \\
\text { Donja strana }\end{array}$ & $\begin{array}{c}\text { Countermove layer thickness, mm } \\
\text { Debljina furnira na donjoj strani, mm }\end{array}$ \\
\hline Nut (NU) / orah & Nut (NU) / orah & 0.6 \\
\hline Nut (NU) / orah & Alder (AL) / joha & 0.6 \\
\hline Nut (NU) / orah & Spruce (SP) / smreka & 0.6 \\
\hline Nut (NU) / orah & Spruce (SP) / smreka & 1.2 \\
\hline Nut (NU) / orah & Spruce (SP) / smreka & 1.5 \\
\hline Nut (NU) / orah & Beach (BE) / bukva & 0.6 \\
\hline Nut (NU) / orah & Beach (BE) / bukva & 0.9 \\
\hline Nut (NU) / orah & Beach (BE) / bukva & 1.2 \\
\hline Nut (NU) / orah & Beach (BE) / bukva & 1.5 \\
\hline & Countermove foil 85-90 g/m² & - \\
\hline
\end{tabular}


by synthetic resin of emission class $\mathrm{E} 1$ is the basic material. Countermove foils are manufactured in basic weight of 70 to $250 \mathrm{~g} / \mathrm{m}^{2}$ (Zemiar et al., 2009). The reason for creating asymmetrically veneered elements is to use financially less demanding (cheaper) materials on hidden surfaces.

\section{MATERIAL AND METHODS 2. MATERIJAL I METODE}

To determine shape stability, bending strength and modulus of elasticity in bending elements, various combinations of veneers or countermove foils were manufactured under laboratory conditions. To determine relationships between the veneer and countermove foil thickness, two thickness series of beech and spruce veneers were developed. These variants are shown in Tab. 2. From each of the variants, two elements were manufactured with $16 \mathrm{~mm}$ particleboards as the bearing (supporting) part of the elements. The elements were manufactured from particleboards 2800 x $2070 \mathrm{~mm}$.

Properties and composition of urea -formaldehyde (UF) adhesive resin used for gluing:

- solid resin (dry mater content ) $63 \%$

- viscosity $450-1100 \mathrm{mPa} \cdot \mathrm{s}$ at $20^{\circ} \mathrm{C}$

- density1120-1150 kg/m³

Glue was applied by a manual glue applicator, average glue spread being $155 \mathrm{~g} / \mathrm{m}^{2}$. The amount of applied glue was determined by a weight method on check samples. With the countermove foil, the glue spread was $80 \mathrm{~g} / \mathrm{m}^{2}$.

A one-stage press was used for pressing the sets under following parameters:

- pressing time $60 \mathrm{~s} / 1 \mathrm{~mm}$ veneer thickness $+300 \mathrm{~s}$

- pressing temperature $110^{\circ} \mathrm{C}$

- working pressure $0.6 \mathrm{~N} / \mathrm{mm}^{2}$

After pressing, the elements were stored in a stack for balancing the temperature and moisture and for curing the glue. After the air-conditioning, all elements were trimmed to a size of $400 \times 760 \mathrm{~mm}$. This size was subsequently used for cutting test specimens for the determination of bending strength and modulus of elasticity in bending according to the CSN EN 310 standard. Seven test specimens were prepared with the perpendicular direction of fibres and 8 test specimens with the longitudinal direction of fibres of each combination.

The determination of warping (shape stability) was carried out according to the CSN 490148 Standard. To prevent the penetration of water vapours into test specimens, their lateral surfaces were painted with a water-soluble white paint. Thus, porous lateral edges of particleboards were sealed. The measurement of warping was carried out by means of an aluminium lath and digital slide gauge measuring to $0.01 \mathrm{~mm}$. The coordinates of measurement points are presented in Fig. 1.

The principle of the measurement of shape stability is presented in Fig. 2.

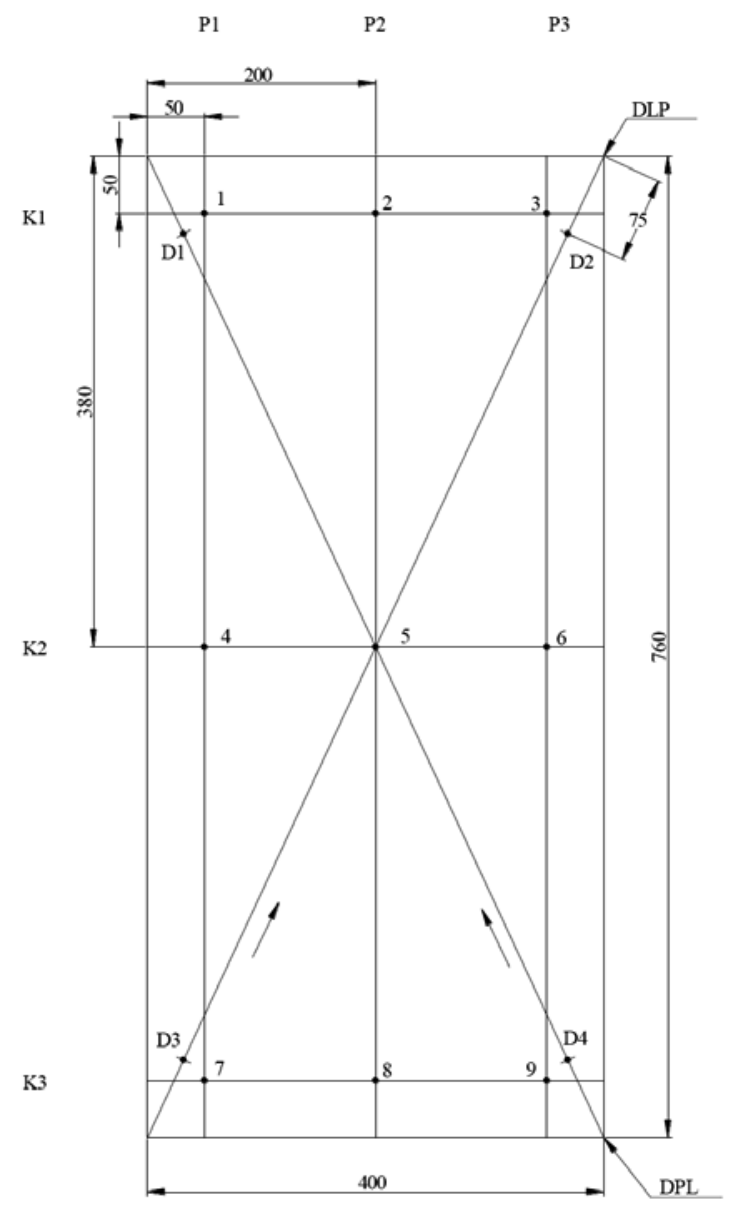

Figure 1 Measurement points of shape stability $(1,2,3,4,5$, 6, 7, 8, 9 - measurement points, D1, D2, D3, D4 - measurement in diagonal direction, P1, P2, P3 - measurement in longitudinal direction, $\mathrm{K} 1, \mathrm{~K} 2, \mathrm{~K} 3$ - measurement in perpendicular direction, DPL - measurement value in diagonal direction - from right to left, DLP5 - measurement in diagonal direction - from left to right)

Slika 1. Mjerne točke stabilnosti oblika $(1,2,3,4,5,6,7,8,9$ - mjerne točke, D1, D2, D3, D4 - mjerenja u dijagonalnom smjeru, P1, P2, P3 - mjerenja u uzdužnom smjeru, K1, K2, K3 - mjerenja u poprečnom smjeru, DPL - mjerna vrijednost $u$ dijagonalnom smjeru - zdesna ulijevo, DLP5 - slijeva udesno)

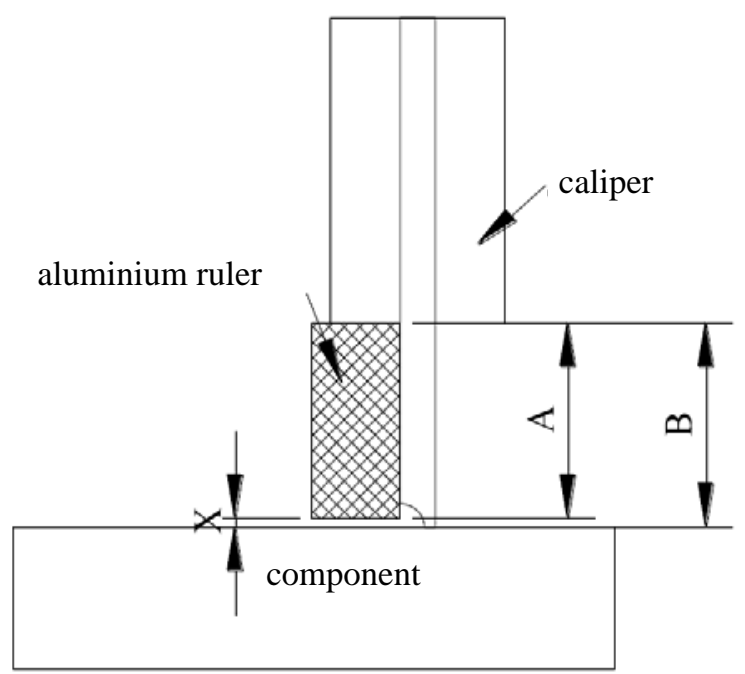

Figure 2 Warping measurement principles Slika 2. Načelo mjerenja izvitoperenosti 
The measurement was carried out on three positions of the ruler. At each position of the ruler, three points were measured on the element/component.

The size of deformation was calculated according to the relation:

$$
X=A-B
$$

Where:

$A$ - aluminium ruler height $22.47 \mathrm{~mm}$

$B$ - value measured by the calliper (depth gauge)

$X$ - deformation

To determine the size of warping corn-wise, the following relation was used

$$
Y=\text { DPL5 }- \text { DLP5 }
$$

Where:

DLP5 - measured value on the diagonal position of the ruler - point 5 (from right to left)

DLP5 - measured value on the diagonal position of the ruler - point 5 (from left to right)

The size of the difference between these values is dependent on the size of warping corn-wise. The larger the difference between diagonal measurements $Y$, the larger is corn-wise warping.

The measurement of warping was carried out before air conditioning and subsequently three times after 7 days of air conditioning. The last measurement was carried out after 6 days of air conditioning. The air conditioning was carried out in a SANYO MTH 2400 climatic chamber. The conditioning regime is presented in Tab. 3.

Air conditioning was carried out with the aim to create stress in surface layers of elements/components causing different levels of warping. The test specimens were placed in the air-conditioning box on the shorter side so as to prevent the development of forces that could prevent warping. Gaps between specific layers were avoided by a locking
Table 3: Air conditioning regime

Tablica 3. Uvjeti kondicioniranja

\begin{tabular}{|c|c|c|}
\hline $\begin{array}{c}\text { Moisture, \% } \\
\text { Vlažnost, \% }\end{array}$ & $\begin{array}{c}\text { Temperature, }{ }^{\circ} \mathbf{C} \\
\text { Temperatura, }{ }^{\circ} \mathrm{C}\end{array}$ & $\begin{array}{c}\text { Time, days } \\
\text { Vrijeme, dani }\end{array}$ \\
\hline $85 \pm 5$ & $30 \pm 2$ & 7 \\
\hline $85 \pm 5$ & $30 \pm 2$ & 7 \\
\hline $30 \pm 5$ & $30 \pm 2$ & 7 \\
\hline $95 \pm 5$ & $30 \pm 2$ & 6 \\
\hline
\end{tabular}

latch, so that changes in gap dimensions could not occur for the period of measurement. According to the CSN 490148 Standard, the warping value in a respective direction is always the highest determined deviation in this direction. It is rounded to $0.1 \mathrm{~mm}$ with 1 meter length $(\mathrm{mm} / \mathrm{m})$.

Bending strength and modulus of elasticity in bending of specific test specimens were determined according to the CSN EN 310 Standard by a ZWICK Z 050 press with 3-point bending.

The measured data were statistically analysed by the STATISTICA version 8 and the Calc 2.0 (OpenOffice). An exploratory data analysis (EDA) was done, assessment of basic characteristics and comparisons were made by Anova, Mann-Whitney U tests, Wald-Wolfowitz tests and Kolmogorov-Smirnov tests.

\section{RESULTS AND DISCUSSION}

3. REZULTATI I RASPRAVA

Measurement and warping calculation were conducted according to the standard CSN 490148. The determined warping values depending on the countermove veneer thickness are given in Tabs. 4 to 7 and in Fig. 3.

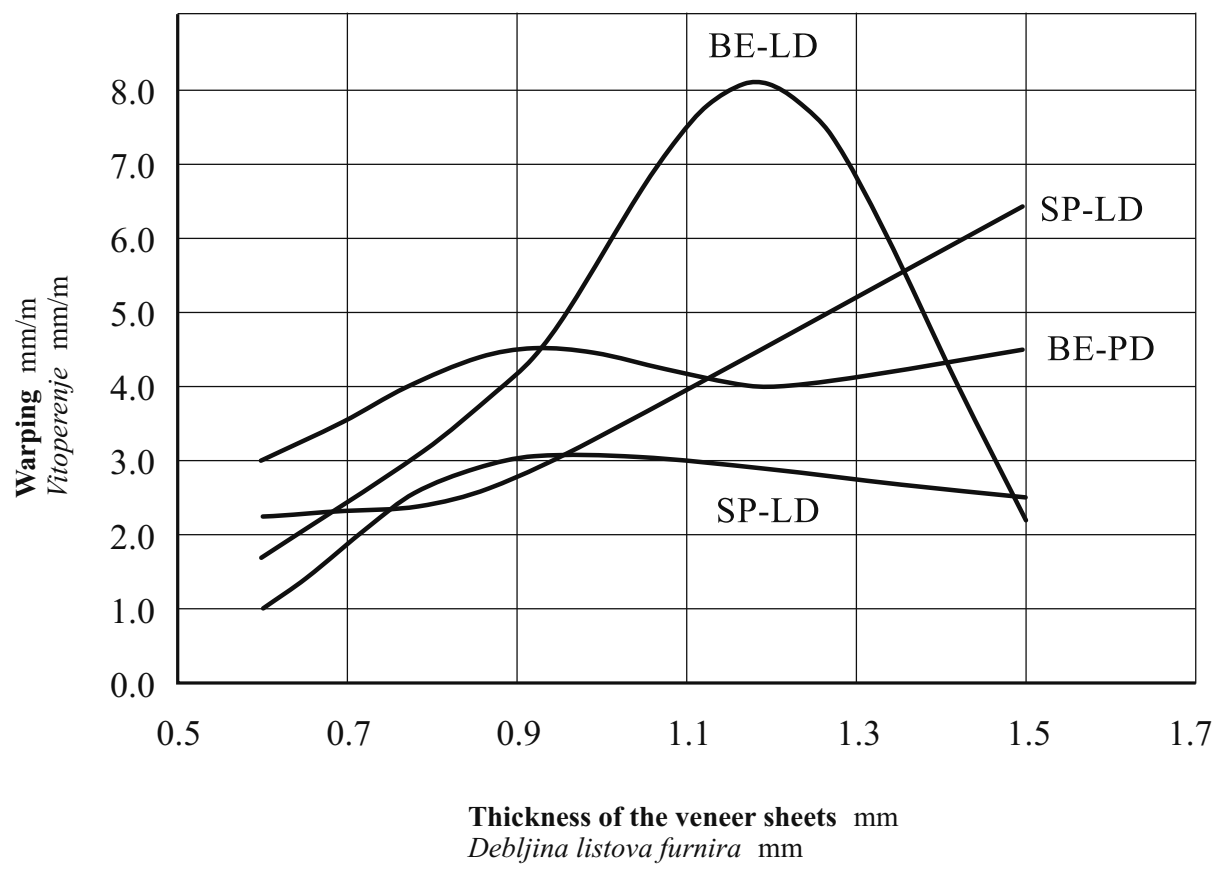

Figure 3 Dependence of warping on countermove veneer thickness

Slika 3. Ovisnost izvitoperenosti o debljini furnira na donjoj strani ploče 
Table 4 Warping values after 14 days of air conditioning Tablica 4. Vrijednosti izvitoperenosti nakon 14 dana kondicioniranja

\begin{tabular}{|c|c|c|}
\hline \multicolumn{3}{|c|}{$\begin{array}{l}\text { Warping values in } \mathbf{m m} / \mathbf{m} \text { after } 14 \text { days of air condi- } \\
\text { tioning } \\
\text { Vrijednosti izvitoperenosti izražene u } \mathrm{mm} / \mathrm{mm} \text { nakon } 14 \\
\text { dana kondicioniranja }\end{array}$} \\
\hline \multirow{2}{*}{$\begin{array}{c}\text { Countermove } \\
\text { layer } \\
\text { Površinska } \\
\text { obrada donje } \\
\text { strane }\end{array}$} & \multicolumn{2}{|c|}{$\begin{array}{l}\text { Direction of measurement } \\
\text { Smjer mjerenja }\end{array}$} \\
\hline & $\begin{array}{l}\text { Longitudinal } \\
\text { Uzdužni } \\
\text { (LD) }\end{array}$ & $\begin{array}{l}\text { Perpendicular } \\
\text { Poprečni } \\
\text { (PD) }\end{array}$ \\
\hline $\mathrm{SP} 0.6 \mathrm{~mm}$ & 2.2 & 1.0 \\
\hline $\mathrm{SP} 1.2 \mathrm{~mm}$ & 2.8 & 3.0 \\
\hline $\mathrm{SP} 1.5 \mathrm{~mm}$ & 6.4 & 2.5 \\
\hline BE $0.6 \mathrm{~mm}$ & 1.7 & 3.0 \\
\hline BE $0.9 \mathrm{~mm}$ & 4.2 & 4.5 \\
\hline $\mathrm{BE} 1.2 \mathrm{~mm}$ & 8.1 & 4.0 \\
\hline $\mathrm{BE} 1.5 \mathrm{~mm}$ & 2.2 & 4.5 \\
\hline
\end{tabular}

Note: SP - spruce / smreka, BE - beech / bukva

Table 6 Warping values after 21 days of air conditioning Tablica 6. Vrijednosti izvitoperenosti nakon 21 dana kondicioniranja

\begin{tabular}{|c|c|c|c|}
\hline \multicolumn{4}{|c|}{$\begin{array}{c}\text { Warping values in mm/m } \\
\text { after 21 days of air conditioning } \\
\text { Vrijednosti izvitoperenosti izražene } u \text { mm/mm } \\
\text { nakon 21 dan kondicioniranja }\end{array}$} \\
\hline $\begin{array}{c}\text { Countermove } \\
\text { layer } \\
\begin{array}{c}\text { Površinska } \\
\text { obrada donje } \\
\text { strane }\end{array}\end{array}$ & $\begin{array}{c}\text { Countermove layer } \\
\text { Površinska obrada donje strane }\end{array}$ \\
\cline { 2 - 4 } & Uzdužno & $\begin{array}{c}\text { Perpendicular } \\
\text { Poprečno }\end{array}$ & $Y$ \\
\hline SP 0.6 mm & 1.1 & 0.5 & 0.2 \\
\hline BE 0.6 mm & 0.3 & 1.0 & 0.2 \\
\hline NU 0.6 mm & 1.7 & 0.5 & 0.3 \\
\hline AL 0.6 mm & 0.8 & 1.5 & 0.2 \\
\hline Foil / folija & 14.4 & 1.5 & 0 \\
\hline
\end{tabular}

Note: SP - spruce / smreka, BE - beech / bukva, NU - walnut / orah, $\mathrm{Ol}$ - alder / joha

Some results (examples) of the measurement of density, bending strength and modulus of rupture in-
Table 5 Evaluation of warping after 14 days of air conditioning

Tablica 5. Procjena izvitoperenosti nakon 14 dana kodicioniranja

\begin{tabular}{|c|c|c|c|}
\hline \multicolumn{3}{|c|}{ Evaluation of warping in mm/m after 14 days of air } \\
conditioning \\
Procjena izvitoperenosti izražene $\begin{array}{c}\text { m mm/mm nakon } 14 \text { dana } \\
\text { kondicioniranja }\end{array}$ \\
\hline $\begin{array}{c}\text { Countermove } \\
\text { layer } \\
\begin{array}{c}\text { Površinska } \\
\text { obrada donje } \\
\text { strane }\end{array}\end{array}$ & $\begin{array}{c}\text { Direction of measurement } \\
\text { Smjer mjerenja }\end{array}$ \\
\cline { 2 - 4 } & Uzdužni & $\begin{array}{c}\text { Perpendicular } \\
\text { Poprečni }\end{array}$ & $Y$ \\
\hline SP 0.6 mm & 2.2 & 1.0 & 1.0 \\
\hline BE $0.6 \mathrm{~mm}$ & 1.7 & 3.0 & 0.7 \\
\hline NU 0.6 mm & 1.9 & 1.5 & 0.4 \\
\hline AL $0.6 \mathrm{~mm}$ & 1.7 & 2.5 & 0.1 \\
\hline Foil / folija & 26.4 & 1.5 & 0 \\
\hline
\end{tabular}

Note: SP - spruce / smreka, BE - beech / bukva, NU - walnut / orah, $\mathrm{AL}$ - alder / joha

Table 7 Warping values after 27 days of air conditioning Tablica 7. Vrijednosti izvitoperenosti nakon 27 dana kondicioniranja

\begin{tabular}{|c|c|c|c|}
\hline \multicolumn{4}{|c|}{$\begin{array}{c}\text { Warping values in mm/m } \\
\text { after 27 days of air conditioning } \\
\text { Vrijednosti izvitoperenosti izražene u } \mathrm{mm} / \mathrm{mm} \\
\text { nakon } 27 \text { dana kondicioniranja }\end{array}$} \\
\hline $\begin{array}{c}\text { Countermove } \\
\text { layer } \\
\begin{array}{c}\text { Površinska } \\
\text { obrada donje } \\
\text { strane }\end{array}\end{array}$ & $\begin{array}{c}\text { Lonntermove layer } \\
\text { Površinska obrada donje strane }\end{array}$ \\
\cline { 2 - 4 } & Uzdužno & $\begin{array}{c}\text { Perpendicular } \\
\text { Poprečno }\end{array}$ & $Y$ \\
\hline BP 0.6 mm $0.6 \mathrm{~mm}$ & 2.8 & 1.5 & 0.7 \\
\hline NU $0.6 \mathrm{~mm}$ & 1.7 & 2.0 & 0.3 \\
\hline AL $0.6 \mathrm{~mm}$ & 1.4 & 1.0 & 0.4 \\
\hline Foil / folija & 26.4 & 2.0 & 0.3 \\
\hline
\end{tabular}

Note: SP - spruce / smreka, BE - beech / bukva, NU - walnut / orah, $\mathrm{AL}$ - alder / joha

cluding characteristics of descriptive statistics are given in Tabs. 8 to 13.

Table 8 Values of density, modulus of elasticity in bending $(M O E)$ and modulus of rupture $(M O R)$

Tablica 8. Vrijednosti gustoće, modula elastičnosti pri savijanju $(M O E)$ i modula loma $(M O R)$

\begin{tabular}{|c|c|c|c|c|c|c|c|c|c|c|}
\hline \multicolumn{7}{|c|}{ Countermove layer - walnut (NU) 0.6 mm / Površinska obrada donje strane - orah (NU) 0,6 mm } \\
\hline & \multicolumn{9}{|c|}{$\begin{array}{c}\text { Longitudinal direction of fibres } \\
\text { Uzdužni smjer vlakanaca }\end{array}$} \\
\hline $\begin{array}{c}\text { Stat. } \\
\text { value }\end{array}$ & $\begin{array}{c}F_{\max } \\
\mathrm{N}\end{array}$ & $\begin{array}{c}M O R \\
\mathrm{~N} / \mathrm{mm}^{2}\end{array}$ & $\begin{array}{c}M O E \\
\mathrm{~N} / \mathrm{mm}^{2}\end{array}$ & $\begin{array}{c}\varepsilon \\
\mathrm{mm}\end{array}$ & $\begin{array}{c}\rho \\
\mathrm{kg} / \mathrm{m}^{3}\end{array}$ & $\begin{array}{c}F_{\max } \\
\mathrm{N}\end{array}$ & $\begin{array}{c}\text { MOR } \\
\mathrm{N} / \mathrm{mm}^{2}\end{array}$ & $\begin{array}{c}\text { MOE } \\
\mathrm{N} / \mathrm{mm}^{2}\end{array}$ & $\begin{array}{c}\varepsilon \\
\mathrm{mm}\end{array}$ & $\begin{array}{c}\rho \\
\mathrm{kg} / \mathrm{m}^{3}\end{array}$ \\
\hline $\mathrm{n}$ & 8 & 8 & 8 & 8 & 8 & 7 & 7 & 7 & 7 & 7 \\
\hline $\bar{x}$ & 729.94 & 26.92 & 3437.9 & 11.92 & 633.6 & 361.33 & 13.22 & 2579.79 & 6.97 & 650.57 \\
\hline $\mathrm{s}$ & 51.98 & 1.95 & 80.12 & 1.19 & 2.83 & 33.66 & 1.21 & 72.82 & 0.7 & 9.98 \\
\hline$V(\%)$ & 7.12 & 7.23 & 2.33 & 9.96 & 0.45 & 9.3 & 9.17 & 2.82 & 10.05 & 1.50 \\
\hline Min. & 644.06 & 23.76 & 3303.29 & 9.99 & 630.0 & 321.9 & 11.8 & 2454.63 & 6.06 & 639.0 \\
\hline Max. & 786.03 & 29.18 & 3548.83 & 13.62 & 639.0 & 409.41 & 14.92 & 2682.63 & 7.71 & 664.0 \\
\hline
\end{tabular}


Table 9 Values of density, modulus of elasticity in bending $(M O E)$ and modulus of rupture (MOR)

Tablica 9. Vrijednosti gustoće, modula elastičnosti pri savijanju $(M O E)$ i modula loma $(M O R)$

\begin{tabular}{|c|c|c|c|c|c|c|c|c|c|c|}
\hline \multicolumn{7}{|c|}{ Countermove layer - foil / Površinska obrada donje strane - folija } \\
\hline & \multicolumn{9}{|c|}{$\begin{array}{c}\text { Longitudinal direction of fibres } \\
\text { Uzdužni smjer vlakanaca } \\
\text { Poprečni smjer vlakanaca }\end{array}$} \\
\hline $\begin{array}{c}\text { Stat. } \\
\text { value }\end{array}$ & $\begin{array}{c}F_{\max } \\
\mathrm{N}\end{array}$ & $\begin{array}{c}\text { MOR } \\
\mathrm{N} / \mathrm{mm}^{2}\end{array}$ & $\begin{array}{c}F_{\max } \\
\mathrm{N}\end{array}$ & $\begin{array}{c}M O R \\
\mathrm{~N} / \mathrm{mm}^{2}\end{array}$ & $\begin{array}{c}F_{\max } \\
\mathrm{N}\end{array}$ & $\begin{array}{c}\text { MOR } \\
\mathrm{N} / \mathrm{mm}^{2}\end{array}$ & $\begin{array}{c}F_{\max } \\
\mathrm{N}\end{array}$ & $\begin{array}{c}\text { MOR } \\
\mathrm{N} / \mathrm{mm}^{2}\end{array}$ & $\begin{array}{c}F_{\max } \\
\mathrm{N}\end{array}$ & $\begin{array}{c}\text { MOR } \\
\mathrm{N} / \mathrm{mm}^{2}\end{array}$ \\
\hline$n$ & 8 & 8 & 8 & 8 & 8 & 7 & 7 & 7 & 7 & 7 \\
\hline $\bar{x}$ & 427.84 & 16.65 & 3091.6 & 7.83 & 636.4 & 407.32 & 15.95 & 2922.77 & 7.94 & 631.57 \\
\hline$s$ & 13.13 & 0.5 & 70.89 & 0.44 & 7.44 & 13.48 & 0.6 & 67.93 & 0.31 & 4.39 \\
\hline$V(\%)$ & 3.1 & 2.98 & 2.29 & 5.55 & 1.17 & 3.31 & 3.75 & 2.32 & 3.91 & 0.70 \\
\hline Min. & 415.57 & 16.17 & 2971.8 & 7.48 & 620.0 & 394.034 & 15.39 & 2861.45 & 7.60 & 625.0 \\
\hline Max. & 446.57 & 17.5 & 3203.0 & 8.71 & 644.0 & 430.39 & 17.01 & 3017.28 & 8.46 & 637.0 \\
\hline
\end{tabular}

Table 10 Values of density, modulus of elasticity in bending $(M O E)$ and modulus of rupture (MOR)

Tablica 10. Vrijednosti gustoće, modula elastičnosti pri savijanju $(M O E)$ i modula loma $(M O R)$

\begin{tabular}{|c|c|c|c|c|c|c|c|c|c|c|}
\hline \multicolumn{7}{|c|}{ Countermove layer - spruce (SP) 0.6 mm / Površinska obrada donje strane - smreka 0,6 mm } \\
\hline & \multicolumn{5}{|c|}{$\begin{array}{c}\text { Longitudinal direction of fibres } \\
\text { Uzdužni smjer vlakanaca }\end{array}$} \\
\hline $\begin{array}{c}\text { Stat. } \\
\text { value }\end{array}$ & $\begin{array}{c}F_{\max } \\
\mathrm{N}\end{array}$ & $\begin{array}{c}\text { MOR } \\
\mathrm{N} / \mathrm{mm}^{2}\end{array}$ & $\begin{array}{c}F_{\max } \\
\mathrm{N}\end{array}$ & $\begin{array}{c}\text { MOR } \\
\mathrm{N} / \mathrm{mm}^{2}\end{array}$ & $\begin{array}{c}F_{\max } \\
\mathrm{N}\end{array}$ & $\begin{array}{c}\text { MOR } \\
\mathrm{N} / \mathrm{mm}^{2}\end{array}$ & $\begin{array}{c}F_{\max } \\
\mathrm{N}\end{array}$ & $\begin{array}{c}\text { MOR } \\
\mathrm{N} / \mathrm{mm}^{2}\end{array}$ & $\begin{array}{c}F_{\max } \\
\mathrm{N}\end{array}$ & $\begin{array}{c}\text { MOR } \\
\text { N/mm } \mathrm{mm}^{2}\end{array}$ \\
\hline$n$ & 8 & 8 & 8 & 8 & 8 & 7 & 7 & 7 & 7 & 7 \\
\hline $\bar{x}$ & 736.57 & 26.72 & 3511.94 & 11.41 & 629.5 & 383.29 & 13.86 & 2505.45 & 7.61 & 629.43 \\
\hline$s$ & 84.08 & 2.99 & 116.5 & 1.8 & 2.07 & 11.8 & 0.44 & 15.75 & 0.35 & 3.41 \\
\hline$V(\%)$ & 11.42 & 11.21 & 3.32 & 16.1 & 0.33 & 3.08 & 3.16 & 0.63 & 4.6 & 0.54 \\
\hline Min. & 591.69 & 21.66 & 3379.72 & 8.5 & 627.0 & 367.54 & 13.28 & 2482.69 & 7.16 & 625.0 \\
\hline Max. & 811.13 & 29.39 & 3713.13 & 13.11 & 634.0 & 399.8 & 14.49 & 2525.67 & 8.15 & 634.0 \\
\hline
\end{tabular}

Table 11 Values of density, modulus of elasticity in bending $(M O E)$ and modulus of rupture $(M O R)$

Tablica 11. Vrijednosti gustoće, modula elastičnosti pri savijanju $(M O E)$ i modula loma $(M O R)$

\begin{tabular}{|c|c|c|c|c|c|c|c|c|c|c|}
\hline \multicolumn{7}{|c|}{ Countermove layer - beech (BE) 0.6 mm / Površinska obrada donje strane - bukva 0,6 mm } \\
\hline & \multicolumn{5}{|c|}{$\begin{array}{c}\text { Longitudinal direction of fibres } \\
\text { Uzdužni smjer vlakanaca }\end{array}$} \\
\hline $\begin{array}{c}\text { Stat. } \\
\text { value }\end{array}$ & $\begin{array}{c}F_{\max } \\
\mathrm{N}\end{array}$ & $\begin{array}{c}M O R \\
\mathrm{~N} / \mathrm{mm}^{2}\end{array}$ & $\begin{array}{c}F_{\max } \\
\mathrm{N}\end{array}$ & $\begin{array}{c}M O R \\
\mathrm{~N} / \mathrm{mm}^{2}\end{array}$ & $\begin{array}{c}F_{\max } \\
\mathrm{N}\end{array}$ & $\begin{array}{c}\text { MOR } \\
\mathrm{N} / \mathrm{mm}^{2}\end{array}$ & $\begin{array}{c}F_{\max } \\
\mathrm{N}\end{array}$ & $\begin{array}{c}\text { MOR } \\
\mathrm{N} / \mathrm{mm}^{2}\end{array}$ & $\begin{array}{c}F_{\max } \\
\mathrm{N}\end{array}$ & $\begin{array}{c}\text { MOR } \\
\mathrm{N} / \mathrm{mm}^{2}\end{array}$ \\
\hline$n$ & 8 & 8 & 8 & 8 & 8 & 7 & 7 & 7 & 7 & 7 \\
\hline $\bar{x}$ & 889.82 & 32.51 & 3819.06 & 13.44 & 642.5 & 394.5 & 14.54 & 2609.9 & 7.72 & 637.29 \\
\hline$s$ & 78.09 & 2.94 & 218.87 & 1.36 & 3.3 & 16.8 & 0.6 & 48.79 & 0.62 & 11.7 \\
\hline$V(\%)$ & 8.78 & 9.06 & 5.65 & 10.08 & 0.51 & 4.26 & 4.15 & 1.87 & 8.07 & 1.84 \\
\hline Min. & 798.37 & 29.03 & 3525.32 & 11.88 & 639.0 & 370.42 & 13.66 & 2542.57 & 6.83 & 624.0 \\
\hline Max. & 1029.43 & 37.7 & 4205.61 & 15.71 & 648.0 & 424.45 & 15.61 & 2680.01 & 8.75 & 657.0 \\
\hline
\end{tabular}

Table 12 Values of density, modulus of elasticity in bending $(M O E)$ and modulus of rupture (MOR)

Tablica 12. Vrijednosti gustoće, modula elastičnosti pri savijanju $(M O E)$ i modula loma $(M O R)$

\begin{tabular}{|c|c|c|c|c|c|c|c|c|c|c|}
\hline \multicolumn{7}{|c|}{ Countermove layer - beech (BE) 1.2 mm / Povř̌inska obrada donje strane-bukva 1,2 mm } \\
\hline & \multicolumn{9}{|c|}{$\begin{array}{c}\text { Lerpendicular direction of fibres } \\
\text { Poprečni smjer vlakanaca }\end{array}$} \\
\hline $\begin{array}{c}\text { Stat. } \\
\text { value }\end{array}$ & $\begin{array}{c}F_{\max } \\
\mathrm{N}\end{array}$ & $\begin{array}{c}\text { MOR } \\
\mathrm{N} / \mathrm{mm}^{2}\end{array}$ & $\begin{array}{c}F_{\max } \\
\mathrm{N}\end{array}$ & $\begin{array}{c}\text { MOR } \\
\mathrm{N} / \mathrm{mm}^{2}\end{array}$ & $\begin{array}{c}F_{\max } \\
\mathrm{N}\end{array}$ & $\begin{array}{c}\text { MOR } \\
\mathrm{N} / \mathrm{mm}^{2}\end{array}$ & $\begin{array}{c}F_{\max } \\
\mathrm{N}\end{array}$ & $\begin{array}{c}\text { MOR } \\
\mathrm{N} / \mathrm{mm}^{2}\end{array}$ & $\begin{array}{c}F_{\max } \\
\mathrm{N}\end{array}$ & $\begin{array}{c}\text { MOR } \\
\mathrm{N} / \mathrm{mm}^{2}\end{array}$ \\
\hline$n$ & 8 & 8 & 8 & 8 & 8 & 7 & 7 & 7 & 7 & 7 \\
\hline $\bar{x}$ & 1293.34 & 43.86 & 5569.99 & 12.21 & 666.38 & 252.52 & 8.5 & 1960.75 & 5.23 & 665.0 \\
\hline$s$ & 205.22 & 6.84 & 318.78 & 2.18 & 13.84 & 19.5 & 0.66 & 37.2 & 0.46 & 1.73 \\
\hline$V(\%)$ & 15.87 & 15.59 & 5.72 & 17.82 & 2.08 & 7.72 & 7.8 & 1.9 & 8.78 & 0.26 \\
\hline Min. & 814.28 & 27.93 & 5130.73 & 6.92 & 646.0 & 231.07 & 7.77 & 1905.58 & 4.68 & 663.0 \\
\hline Max. & 1472.94 & 50.10 & 6054.73 & 13.65 & 688.0 & 277.23 & 9.36 & 2007.39 & 5.81 & 668.0 \\
\hline
\end{tabular}


.... Král, Hrázský, Hrapková, Hamšík: Shape stability of particleboards surface-treated by ...

Table 13 Values of density, modulus of elasticity in bending $(M O E)$ and modulus of rupture (MOR)

Tablica 13. Vrijednosti gustoće, modula elastičnosti pri savijanju (MOE) i modula loma $(M O R)$

\begin{tabular}{|c|c|c|c|c|c|c|c|c|c|c|}
\hline \multicolumn{11}{|c|}{ Countermove layer - alder (AL) $\mathbf{0 . 6} \mathbf{~ m m ~ / ~ P o v r s ̌ i n s k a ~ o b r a d a ~ d o n j e ~ s t r a n e ~ - ~ j o h a ~ 0 , 6 ~ m m ~}$} \\
\hline & \multicolumn{5}{|c|}{$\begin{array}{l}\text { Longitudinal direction of fibres } \\
\text { Uzdužni smjer vlakanaca }\end{array}$} & \multicolumn{5}{|c|}{$\begin{array}{c}\text { Perpendicular direction of fibres } \\
\text { Poprečni smjer vlakanaca }\end{array}$} \\
\hline $\begin{array}{l}\text { Stat. } \\
\text { value }\end{array}$ & $F_{\max }$ & $\begin{array}{c}M O R \\
\mathrm{~N} / \mathrm{mm}^{2}\end{array}$ & $F_{\max }$ & $\begin{array}{c}M O R \\
\mathrm{~N} / \mathrm{mm}^{2}\end{array}$ & $F_{\max }$ & $\begin{array}{c}M O R \\
\mathrm{~N} / \mathrm{mm}^{2}\end{array}$ & $F_{\max }$ & $\begin{array}{c}M O R \\
\mathrm{~N} / \mathrm{mm}^{2}\end{array}$ & $F_{\max }$ & $\begin{array}{c}M O R \\
\mathrm{~N} / \mathrm{mm}^{2}\end{array}$ \\
\hline$n$ & 8 & 8 & 8 & 8 & 8 & 7 & 7 & 7 & 7 & 7 \\
\hline $\bar{x}$ & 821.27 & 30.08 & 4201.24 & 10.71 & 643.0 & 285.63 & 10.46 & 1965.57 & 7.09 & 641.57 \\
\hline$s$ & 48.8 & 1.78 & 166.79 & 1.0 & 10.01 & 19.18 & 0.72 & 19.46 & 0.68 & 2.7 \\
\hline$V(\%)$ & 5.94 & 5.91 & 3.97 & 9.36 & 1.56 & 6.72 & 6.92 & 0.99 & 9.63 & 0.4 \\
\hline Min. & 743.38 & 27.29 & 3953.57 & 8.74 & 633.0 & 259.22 & 9.46 & 1936.0 & 6.17 & 638.0 \\
\hline Max. & 890.16 & 32.57 & 4447.75 & 11.7 & 661.0 & 313.59 & 11.55 & 1993.18 & 8.05 & 645.0 \\
\hline
\end{tabular}

The longest period of air conditioning with constant parameters (according to Tab. 3) was 14 days. The measured warping values of veneered particleboards (different variants) are summarized in Tab. 4. According to values given in the table, it is evident that warping of individual elements in longitudinal direction increases with the thickness of the countermove spruce veneer. The highest values were found with spruce veneer, $1.5 \mathrm{~mm}$ thick $(6.4 \mathrm{~mm} / \mathrm{m})$, and also with beech veneer, $1.2 \mathrm{~mm}$ thick $(8.1 \mathrm{~mm} / \mathrm{m})$. With the use of a countermove beech veneer, $1.5 \mathrm{~mm}$ thick, the warping value dropped to $2.2 \mathrm{~mm} / \mathrm{m}$. In perpendicular direction, the highest warping values were determined with the use of a countermove spruce (SP) veneer, $1.2 \mathrm{~mm}$ thick $(3.0 \mathrm{~mm} / \mathrm{m})$, and beech (BE), 0.9 $\mathrm{mm}$ in thick $(4.5 \mathrm{~mm} / \mathrm{m})$. A variant with the use of a countermove walnut (NU) veneer, $0.6 \mathrm{~mm}$ thick, was selected as a reference variant to compare the warping values. It refers to a symmetric variant, i.e. a walnut (NU) veneer, $0.6 \mathrm{~mm}$ thick, was also used on the tight side of the particleboard. Evaluations of $P, K$ and $Y$ warping of individual variants were compared with warping values of this reference variant.

Tab. 5 presents the warping values of the elements after a 14-day air conditioning. It follows that alder (AL), $0.6 \mathrm{~mm}$ thick, appears to be the most suitable countermove. An element (component) veneered in this way shows lower warping values in longitudinal direction $(1.7 \mathrm{~mm} / \mathrm{m})$ and low values of $Y(0.1$ $\mathrm{mm} / \mathrm{m}$ ), which expresses the level of corn-wise warping. In perpendicular direction, however, a higher warping value was determined $(2.5 \mathrm{~mm} / \mathrm{m})$.
According to Tab. 6 (air conditioning for 21 days), an element veneered on the underside with a countermove beech (BE) veneer, $0.6 \mathrm{~mm}$ thick, showed the lowest warping values. In the longitudinal direction, warping of $0.3 \mathrm{~mm} / \mathrm{m}$ was found, while 1.0 $\mathrm{mm} / \mathrm{m}$ was found in perpendicular direction and the relative $Y$ value expressing corn-wise warping was 0.2 $\mathrm{mm} / \mathrm{m}$.

According to Tab. 7 that presents the warping values after 27 days of air conditioning, an alder (AL) veneer, $0.6 \mathrm{~mm}$ thick, appears to be the most suitable countermove material. In longitudinal direction, warping of $1.4 \mathrm{~mm} / \mathrm{m}$ was determined, while $2.0 \mathrm{~m} / \mathrm{m}$ was found in perpendicular direction and the $Y$ value expressing corn-wise warping was $0.3 \mathrm{~mm} / \mathrm{m}$.

With the use of a countermove foil, surprisingly high warping values were found in longitudinal direction. After a 14-day air conditioning of elements, these values were up to 13.89 times higher than those of the reference symmetrically veneered element. In our opinion, the cause of warping was the low surface density of countermove foil.

Dependence between sheathing materials and values of $M O R$ and $M O E$ of veneered materials warping values and $M O E$ and $M O R$ were also compared in both longitudinal and perpendicular directions. Statistical evaluation of the modulus of elasticity of test specimens with the longitudinal direction of fibres $(P)$ is presented in Tabs. 14 to 15 . First, the one-dimensional test of significance was carried out (Tab. 14), and then the Tuckey HSD test of multiple comparisons (Tab. 15).

Table 14 One-dimensional test of significance for MOE-P (longitudinal direction)

Tablica 14. Jednodimenzionalni test značajnosti MOE-P (uzdužni smjer)

\begin{tabular}{|c|c|c|c|c|c|}
\hline \multirow{2}{*}{ Effect } & \multicolumn{5}{|c|}{$\begin{array}{c}\text { One-dimensional tests of significance for MOE - P } \\
\text { Sigma-limited parameterisation } \\
\text { Decomposition of an effective hypothesis }\end{array}$} \\
\cline { 2 - 6 } & Value of variance & Degree of freedom & $\begin{array}{c}\text { Value of variance } \\
\text { effect of the factor }\end{array}$ & $F$ & $p$ \\
\hline Absolute term & $1.392855 \mathrm{E}+09$ & 1 & $1.392855 \mathrm{E}+09$ & 40803.48 & 0.00 \\
\hline Combination & $4.804689 \mathrm{E}+07$ & 9 & $5.338543 \mathrm{E}+06$ & 156.39 & 0.00 \\
\hline Error & $2.355363 \mathrm{E}+06$ & 69 & $3.413570 \mathrm{E}+04$ & & \\
\hline
\end{tabular}




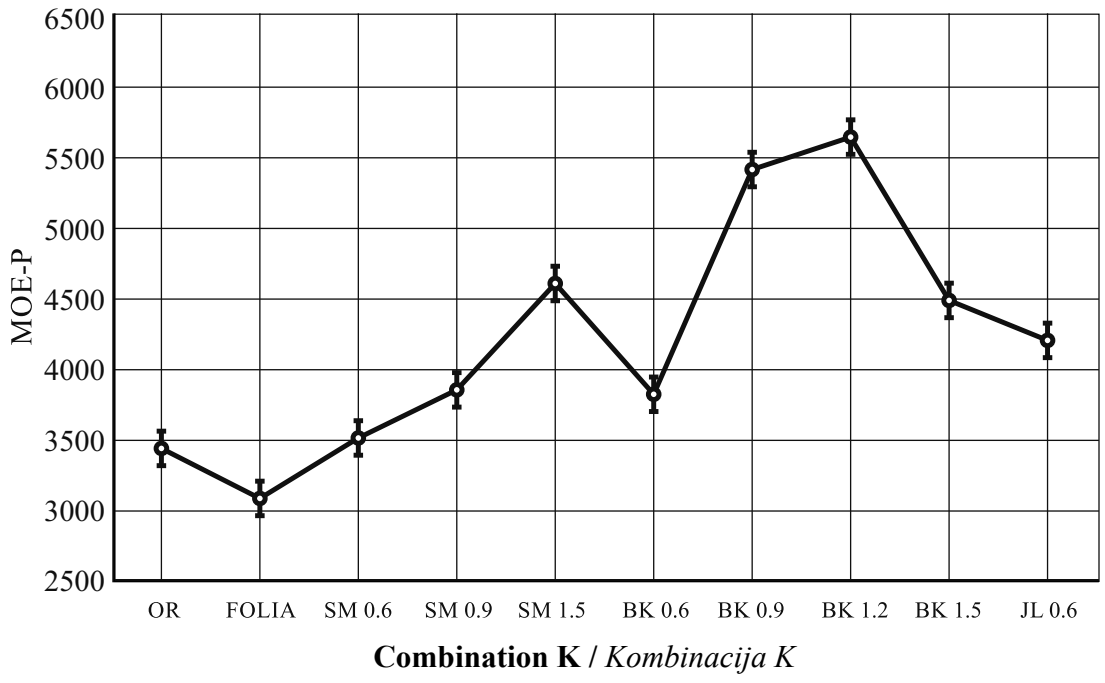

Note: OR - Walnut, Foil, $\mathrm{SP}$ - spruce, $\mathrm{BK}$ - beech, JL - elm, OL - alder Legenda: OR - orah, folija, SP . smreka, BK-bukva, JL-brijest, OL - joha

Figure 4 Relationship between mean values of MOE-P

Slika 4. Prikaz srednjih vrijednosti MOE-P

The value of $p$ is smaller than the value of $\alpha$, which implies that the assessed factor (combination) shows a statistically significant effect on the measured value. In this case, tests of multiple comparisons were subsequently made (Tab. 15). The relationship between mean values of MOE-P is shown in Fig. 4.

Table 15 Tuckey's HSD test of multiple comparisons Tablica 15. Tuckeyev HSD test višekratnih usporedbi

\begin{tabular}{|c|c|c|c|c|c|c|c|c|c|c|c|}
\hline 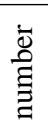 & & & & $\begin{array}{r}\mathrm{T} \imath \\
\text { Appr } \\
\text { Erro } \\
\end{array}$ & $\begin{array}{l}\text { ckey's HS } \\
\text { oximate pr } \\
\text { r: intergrou }\end{array}$ & $\begin{array}{l}\text { test; varia } \\
\text { obabilities } \\
\text { p PČ }=341\end{array}$ & $\begin{array}{l}\text { ible MOE } \\
\text { for post ho } \\
36 ; \mathrm{sv}=6\end{array}$ & $\begin{array}{l}-\mathrm{P} \\
\mathrm{c} \text { tests } \\
.000\end{array}$ & & & \\
\hline$\overline{\bar{\theta}}$ & $\begin{array}{l}\text { Kombi- } \\
\text { nace }\end{array}$ & $\begin{array}{c}\{1\} \\
3437.9\end{array}$ & $\begin{array}{c}\{2\} \\
3091.6\end{array}$ & $\begin{array}{c}\{3\} \\
3511.9\end{array}$ & $\begin{array}{c}\{4\} \\
3851.6 \\
\end{array}$ & $\begin{array}{c}\{5\} \\
4591.9 \\
\end{array}$ & $\begin{array}{c}\{6\} \\
3819.1\end{array}$ & $\begin{array}{c}7\} \\
5393.3 \\
\end{array}$ & $\begin{array}{c}\{8\} \\
5632.9\end{array}$ & $\begin{array}{c}\{9\} \\
4491.9\end{array}$ & $\begin{array}{c}\{10\} \\
4201.1\end{array}$ \\
\hline 1 & NU & & 0.012710 & 0.998398 & 0.001249 & 0.000164 & 0.003870 & 0.000164 & 0.000164 & 0.000164 & 0.000164 \\
\hline 2 & FOLIA & 0.012710 & & 0.001004 & 0.000164 & 0.000164 & 0.000164 & 0.000164 & 0.000164 & 0.000164 & 0.000164 \\
\hline 3 & SP 0.6 & 0.998398 & 0.001004 & & 0.015720 & 0.000164 & 0.043010 & 0.000164 & 0.000164 & 0.000164 & 0.000164 \\
\hline 4 & SP 0.9 & 0.001249 & 0.000164 & 0.015720 & & 0.000164 & 0.999998 & 0.000164 & 0.000164 & 0.000164 & 0.011419 \\
\hline 5 & SP 1.5 & 0.000164 & 0.000164 & 0.000164 & 0.000164 & & 0.000164 & 0.000164 & 0.000164 & 0.984929 & 0.002784 \\
\hline 6 & BE 0.6 & 0.003870 & 0.000164 & 0.043010 & 0.999998 & 0.000164 & & 0.000164 & 0.000164 & 0.000164 & 0.003771 \\
\hline 7 & BE 0.9 & 0.000164 & 0.000164 & 0.000164 & 0.000164 & 0.000164 & 0.000164 & & 0.283974 & 0.000164 & 0.000164 \\
\hline 8 & BE 1.2 & 0.000164 & 0.000164 & 0.000164 & 0.000164 & 0.000164 & 0.000164 & 0.283974 & & 0.000164 & 0.000164 \\
\hline 9 & BE 1.5 & 0.000164 & 0.000164 & 0.000164 & 0.000164 & 0.984929 & 0.000164 & 0.000164 & 0.000164 & & 0.068853 \\
\hline 10 & AL 0.6 & 0.000164 & 0.000164 & 0.000164 & 0.011419 & 0.002784 & 0.003771 & 0.000164 & 0.000164 & 0.068853 & \\
\hline
\end{tabular}

Note: NU - Walnut / orah, Foil / folija, SP - spruce / smreka, BE - beech / bukva, AL - alder / joha

Statistical evaluation of the modulus of elasticity of test specimens with the perpendicular direction of fibres $(K)$ is shown in Tabs. 16 to 17. First, one-dimensional test of significance was carried out (Tab. 16), and subsequently the Tuckey's HSD test of multiple comparison (Tab. 17). The relationship between mean values is shown in Fig. 5. Test specimens with countermove spruce (SP) of 1.5 layer and beech (BE) of 1.2 layer were excluded from the evaluation, on the ground of the countermove veneer disturbance in the gap of the glued veneer sheets. The relationship between mean values of MOE-K is shown in Fig. 5. 
Table 16 One-dimensional test of significance for MOE - K (perpendicular direction)

Tablica 16. Jednodimenzionalni test značajnosti MOE-K (poprečni smjer)

\begin{tabular}{|c|c|c|c|c|c|}
\hline \multirow{2}{*}{ Effect } & \multicolumn{5}{|c|}{$\begin{array}{c}\text { One-dimensional tests of significance for MOE - K } \\
\text { Sigma-limited parameterisation } \\
\text { Decomposition of an effective hypothesis }\end{array}$} \\
\cline { 2 - 6 } & SČ & Degree of freedom & PČ & $F$ & $p$ \\
\hline Absolute member & 326768841 & 1 & 326768841 & 123279.6 & 0.00 \\
\hline Combination & 5111581 & 7 & 730226 & 275.5 & 0.00 \\
\hline Error & 127230 & 48 & 2651 & & \\
\hline
\end{tabular}

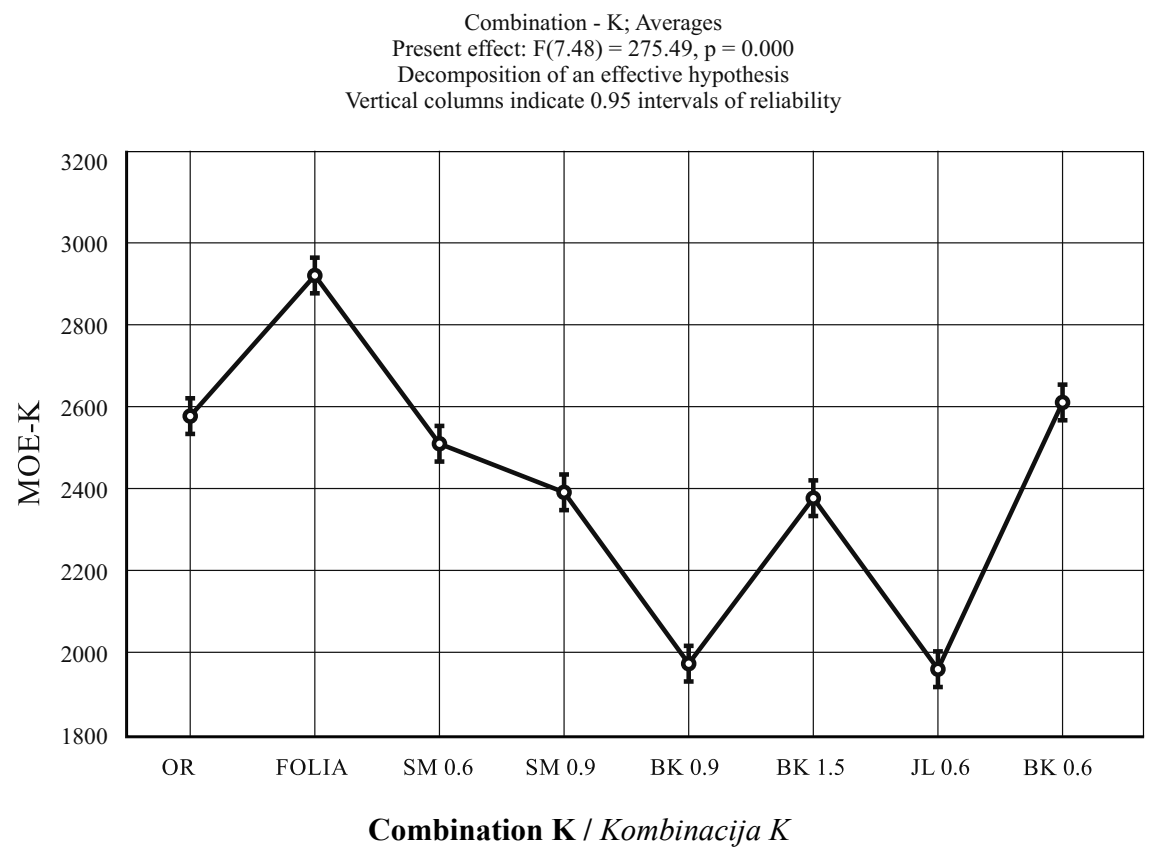

Note: OR - Walnut, Folia - Foil, SM - spruce, BK - beech, JL - elm

Legenda: OR - orah, folija, SM-smreka, BK-bukva, JL-brijest

Figure 5 Relationship between mean values of MOE-K

Slika 5. Prikaz srednjih vrijednosti MOE-K

Table 17 Tuckey's HSD test of multiple comparisons

Tablica 17. Tuckeyev HSD test višekratnih usporedbi

\begin{tabular}{|c|c|c|c|c|c|c|c|c|c|}
\hline \multirow{2}{*}{$\begin{array}{l}\bar{\Xi} \\
\bar{\Xi} \\
\Xi \\
\bar{\Xi}\end{array}$} & \multicolumn{9}{|c|}{$\begin{array}{l}\text { Tuckey's HSD test; variable MOE }-\mathrm{P} \\
\text { Approximate probabilities for post hoc tests } \\
\text { Error: intergroup PC }=2650.6 ; \mathrm{sv}=48.000\end{array}$} \\
\hline & $\begin{array}{l}\text { Combina- } \\
\text { tion }\end{array}$ & $\begin{array}{c}\{1\} \\
2579.9\end{array}$ & $\begin{array}{c}\{2\} \\
2922.6\end{array}$ & $\begin{array}{c}3\} \\
2505.7\end{array}$ & $\begin{array}{c}4\} \\
2392.3\end{array}$ & $\begin{array}{c}5\} \\
1973.3\end{array}$ & $\begin{array}{c}6\} \\
2375.6\end{array}$ & $\begin{array}{c}\{7\} \\
1965.6\end{array}$ & $\begin{array}{c}\{8\} \\
2610.0\end{array}$ \\
\hline 1 & NU & & 0.000134 & 0.149465 & 0.000134 & 0.000134 & 0.000134 & 0.000134 & 0.954778 \\
\hline 2 & FOLIA & 0.000134 & & 0.000134 & 0.000134 & 0.000134 & 0.000134 & 0.000134 & 0.000134 \\
\hline 3 & SP 0.6 & 0.149465 & 0.000134 & & 0.003523 & 0.000134 & 0.000617 & 0.000134 & 0.009347 \\
\hline 4 & SP 0.9 & 0.000134 & 0.000134 & 0.003523 & & 0.000134 & 0.998668 & 0.000134 & 0.000134 \\
\hline 5 & BE 0.9 & 0.000134 & 0.000134 & 0.000134 & 0.000134 & & 0.000134 & 0.999993 & 0.000134 \\
\hline 6 & BE 1.5 & 0.000134 & 0.000134 & 0.000617 & 0.998668 & 0.000134 & & 0.000134 & 0.000134 \\
\hline 7 & OL 0.6 & 0.000134 & 0.000134 & 0.000134 & 0.000134 & 0.999993 & 0.000134 & & 0.000134 \\
\hline 8 & BE 0.6 & 0.954778 & 0.000134 & 0.000134 & 0.000134 & 0.000134 & 0.000134 & 0.000134 & \\
\hline
\end{tabular}

Note: NU - Walnut / orah, Foil / folija, SP - spruce / smreka, BE - beech / bukva, AL - alder / joha 
Tabs. 15 and 17 present $p$ values of the selected test of multiple comparisons for all combinations of selection. If $p$ values $>\alpha$, a hypothesis of the congruence of mean values is accepted, so that values marked black are larger than $\alpha$.

When considering the same relationships between sheathing materials in MOE values of veneered materials and their warping values, it would be easier to find a suitable countermove material and not to test the shape stability (technically and financially rather demanding) of elements (components). On the basis of this consideration, relationships were compared between warping and values of MOE-P and MOE-K. Fig. 3 and data in Tab. 15 clearly show that there is a statistically significant congruence between MOE-P values of an element and countermove layers - walnut (NU) 0.6 and spruce (SP) 0.6. As samples (elements) with a countermove layer - spruce (SP) 0.6 and values MOE-P and MOE-K are significantly consistent with values MOE-P and MOE-K of elements with a countermove layer - walnut (NU) 0.6, the elements where spruce (SP) veneer 0.6 was used as a countermove layer were supposed to achieve the best shape stability. According to Tabs. 5 to 7, however, the lowest warping values are achieved with elements using the countermove layer - alder (AL) $0.6 \mathrm{~mm}$. In Fig. 4, the points representing the mean value of MOE-P show that they would be consistent with curves for longitudinal warping (Fig. 3). It can be concluded that in this case values of MOE and warping correspond. Therefore, it is probable that the difference, caused by the determination of the most suitable material from the aspect of warping values and values of MOE, was the result of a small number of samples in measuring shape stability (warping).

The reason for creating asymmetrically veneered elements is to use financially less demanding (cheaper) materials on hidden surfaces. The function of this material is to provide shape stability.

\section{CONCLUSION}

\section{ZAKLJUČAK}

With increasing demands for decorative veneers, their resources become scarce and their price grows. Increasing efforts to lower production costs force producers to look for possibilities of reducing prices of inputs and searching for new technical solutions. This paper was aimed at searching suitable countermove materials for the underside of veneered elements. One of the tasks of the countermove layer is to provide the shape stability of an element (surface-finished) by veneering. Already in the production stage, it is necessary to eliminate the effects of factors influencing the shape stability of the elements. As asymmetrically veneered elements are more liable to shape changes (warping) than elements veneered symmetrically, it is more suitable to use materials that can minimize the development of moisture differences within an element, such as adhesive foils. At veneering specific elements, the thickness of used veneers has to be always the same. The results of the measurement of warping of the elements veneered by various combinations of veneers and a countermove foil showed that the combination of $0.6 \mathrm{~mm}$ American walnut and $0.6 \mathrm{~mm}$ alder provided the lowest warping values. Considerable savings can be achieved with this combination of materials. In searching for relationships between warping values and $M O E$ values in longitudinal and perpendicular directions, $M O E$ correspondence (congruence) was found with elements using countermove layers - 0.6 mm American walnut and $0.6 \mathrm{~mm}$ spruce.

\section{REFERENCES \\ 5. LITERATURA}

1. Avramidis, G.; Nothnick, H.; Viol, V.; Wolkenhauser, A., 2011: Accelerated curing of PVAC adhesive on plasmatreated wood veneers. European Journal of Wood Products. Volume 69: 329-332.

2. Král, P.; Hrázský, J., 2005: Kompozitní materiály na bázi dřeva. Část II: Dýhy a vrstvené masivni materiály (Composite materials based on wood. Part II: Veneers and laminated solid materials). MZLU v Brně. 210 pp.

3. Požgaj, A.; Chovanec, D.; Kurjatko, S.; Babiak, M., 1997: Štruktúra a vlastnosti dreva (Structure and properties of wood). Vydavatelstvo Príroda Bratislava. 486 pp.

4. Šrajer, J.; Král, P.; Čermák, M.; Mazal, P., 2013: Structure Evaluation of Compressing of Spruce and Beech Plied Boards, Part 1: Microskopic structure, 2013, Wood Research 58: 101-112.

5. Ugolev, V., N., 1975: Drevesinovedenije s osnovami lesnovo tovarovedenia. Moskva.

6. Zemiar, J. et al., 2009: Technológia výroby nábytku (Technology of furniture manufacture). TU Zvolen, 287 pp.

7. *** ČSN EN 310 Desky ze dřeva. Stanoveni modulu pružnosti $v$ ohybu a pevnosti $v$ ohybu (Wood-based panels. Determination of modulus of elasticity in bending and modulus of rupture). ČNI Praha. 1995:8.

8. *** ČSN 490148 Dosky $z$ dreva. Stanovenie šúverenia dosák (Wood-based panels. Determination of warping ). ČNI Praha. 1993:8.

Acknowledgement - Zahvala

Our work was supported by the European Social Fund and the state budget of the Czech Republic, within the project "The Establishment of an International Research Team for the Development of New Wood-based Materials” Reg. No. CZ.1.07/2.3.00/20.0269.

\section{Corresponding address:}

Associate Professor PAVEL KRÁL, Ph.D.

Mendel University

Faculty of Forestry and Wood Technology

Zemědělská 1

61300 Brno, CZECH REPUBLIC

e-mail:kral@mendelu.cz 